\title{
An Integrated Knowledge Base for Modelling and Predicting Vehicle Real-world Emissions as a Function of Driving Behaviour Kinematics
}

\author{
Mario Rapone ${ }^{\dagger}$, Livia Della Ragione and Giovanni Meccariello \\ Istituto Motori CNR, Naples, Italy
}

\begin{abstract}
A multivariate modelling approach was developed by Istituto Motori to model and predict vehicle real-world emissions. Complex driving kinematics is represented by two blocks of variables, which require the development of a hierarchical multiblock emission model, where the two blocks of variables represent overall and instantaneous features of each driving cycle associated to a trip. The multiblock model was applied to analyze and model emissions of the large database built in the ARTEMIS project. In this database we collected emission measurements performed in European laboratories relative to real driving cycles which are statistically representative of many European traffic/road conditions from congested to rush-hour traffic in urban, rural and highway roads. Data concern a varied fleet of vehicles differing in technology and class. To develop a tool useful for mobility analysts for traffic environmental impact assessment, a knowledge base was envisaged to integrate the data warehouse and the model base to build a user interface for driving cycle kinematics and emission analysis. In this paper the modelling approach is presented together with overall emission and driving kinematics characterization based on experimental results, as well as functional analysis of the knowledge base structure and the information tool.
\end{abstract}

Keywords: hierarchical PLS multiblock, kinematic parameters, decision support systems (DSS), real-world emissions, data warehouse

\section{Introduction}

The effect of greenhouse gases on climate has gained global interest and pushed top level policy and decision-makers to promote action for the reduction of emissions.

Kyoto 2008 will define scenarios, policy and strategies for the control of $\mathrm{CO}_{2}$ produced by each country.
Transport activities contribute significantly to air pollutant emissions. Besides $\mathrm{CO}_{2}$,other traffic emissions have a major impact on human health, and for historical towns, on architectural heritage. Evalution of emissions has therefore gained institutional importance in the international community.

Emission evaluation can be performed for different purposes and uses at different space and time scales within trasport envinronmental impact assessment. National, regional, and city emission inventories require overall average emission estimation based on a few input parameters such as fleet composition and average mission profile, average speed related to average traffic situation (Macro scale analysis). Development of regional and city transit plans require detailed representation of the area traffic and transit network into links. For each link average emissions are generally evaluated for each individual vehicle as a function of vehicle flow and average speed (Meso scale analysis). More detailed analysis requires vehicle driving behaviour represented by the time series of vehicle speed on the link, hereinafter called driving cycle (Micro scale). The assessment of the environmental impact of measures regarding road crossing control or development of new infrastructure requires analysis of instantaneous emissions of individual vehicles to estimate the effect of the planned measures on vehicle speed and acceleration. 
The most commonly used macro-scale emission models used in the USA are MOBILE 6 (EPA U.S. Environmental Protection Agency) [1] and EMFAC (CARB California Environmental Protection Agency Air Resources Board) [2]. MOBILE 6 predicts average emission (expressed as $\mathrm{g} / \mathrm{mile}$ ) of regulated pollutants and toxics from LDV, HD, and motorcycles under various conditions, related to in-use emission levels specified by different factors, like ambient temperature, average traffic speed etc. EMFAC predicts emissions with only average trip speed as kinematic input and calculates emission rates both for light duty passenger cars and heavy duty trucks, for highways, freeways and local roads in California. In particular, emission rates are multiplied by vehicle activity data (measured, for example, as miles driven per day) to calculate regional emission inventories. These models work well for large regional areas and they cannot be utilized for macro and meso scales.

In Europe, different meso scale models have been developed. COPERT III and its more recent version COPERT IV, developed in Europe by the LAT (Laboratory of Applied Thermodynamics-Aristotle University) [3], is an average speed model for centralized emission estimation. This model is the official method of the EEA European Environmental Agency for emission calculations and in the new version it takes account of the technology category and future emission standards. Also, in the context of ARTEMIS EU project [4], different models have been developed, classified as traffic situation models and kinematic models. Since 1998, INFRAS (Switzerland) in cooperation with Germany and Austria, has developed the HBEFA Handbook Emission Factors for Road Transport (HBEFA). This model considers kinematic aspects through traffic situations, intended as a combination of road and traffic parameters. The model provides emission factors for all current vehicle categories (PC, LDV, HDV and motor cycles), each divided into different categories, for a wide variety of traffic situations. The latest version is 2.1 and dates from February 2004, in which the emission factors of the HDV (heavy duty vehicles) are completely revised and new measurements for all vehicle categories up to EURO3 are included. The kinematic model approach in ARTEMIS was developed by Rapone [5], and it predicts average emissions on a microtrip (driving pattern) obtaining a detailed de- scription of the velocity profile using a multidimensional approach. This kinematic model (Kinematic Emission Model KEM) is defined as a meso-scale emission model, and it is to be pointed out that it may require less detailed information with respect to instantaneous models. Also, it is based on the analysis of an existing huge emissions database (different fleet composition, different driving sub-cycle and laboratories involved in the emissions measurements), coming from the ARTEMIS EU project.

Within the kinematic approach, the last version of VERSIT+LD [6] is also to be considered. It is a new generation emission factor model based on a multiple regression model, that calculates emission factors for passenger cars as a function of driving cycle characteristics.

In the United States, since 1995 CE-CERT at the University of California-Riverside developed the so-called CMEM Comprehensive Modal Emission Model [7-8-9-10], a modal emission model for light duty vehicles and small trucks. In this model, emissions are calculated as a function of the vehicle operating mode. The CMEM is also able to predict secondby-second emissions and fuel consumption for a wide range of vehicle and technology categories, also for extremely low emitting vehicles [11].

A set of meso and micro scale models has been constructed by the TRL Transport Research Laboratory. The TRL model set evaluates and compares instantaneous emission models [12], PHEM (passenger car parts and heavy duty parts), VeTESS (vehicle transient emission simulation model) and the extended version of MODEM. These models calculate emissions for any vehicle operating profile, but they require detailed and precise information on vehicle operation and also geographical location.

The kinematic model approach developed by CNR-IM was implemented into a decision support system (DSS), useful for mobility analysts for traffic environmental impact assessment. DSS components are the emission data warehouse, the model base and the user interface, for driving cycle kinematics and emission analysis. The DSS builds a knowledge base for modelling and predicting vehicle real-world emissions as a function of driving behavior kinematics. 
In this paper, the DSS structure and the modelling approach are presented, together with some results of the driving behavior kinematics analysis and predicted emission factors of a case study, Euro III gasoline passenger cars in the $1200-1400 \mathrm{cc}$ displacement range.

\section{Information System (IS) Structure}

As a basic requirement, the DSS should contain:

(1) an intuitive and flexible interface;

(2) a guide to problem formulation;

(3) a (possibly automatic) way for choosing the (best) solution method;

(4) a set of rules for sensitivity analysis;

(5) an information retrieval system for previously solved cases;

(6) a (possibly/perhaps automatic) procedure for adapting previously rejected items.

In discussing these points it should be noted that they refer to a DSS for research purposes. However, items (2), (4), and partly (3), are of no interest to the management activity of the DSS. In addition to the above feature and bearing [13] in mind, it would seem useful for us to formulate a "TOOLBOX" principle of a DSS organization [14]. Implementation of this principle means that the DSS should contain a universal set of tools (as for the DSS with research activity, but not to a very great extent) which allows various problems to be solved by a set of users who have different professional requirements. Of course, separate modules for separate problems are required for the principle realization. It is worth noting that the proposed universality of the DSS implies the possibility of different approaches to be used for solving the same problems. Accordingly, the flow diagram (Figure 1) of the DSS may contain parallel branches, and the actual set of modules used to solve a problem should be user-defined. This feature of the DSS makes it necessary to develop rigorous formats of information (input, output and intermediate). Some tools for DSS file handling (archive, update, delete, etc.) are also required.

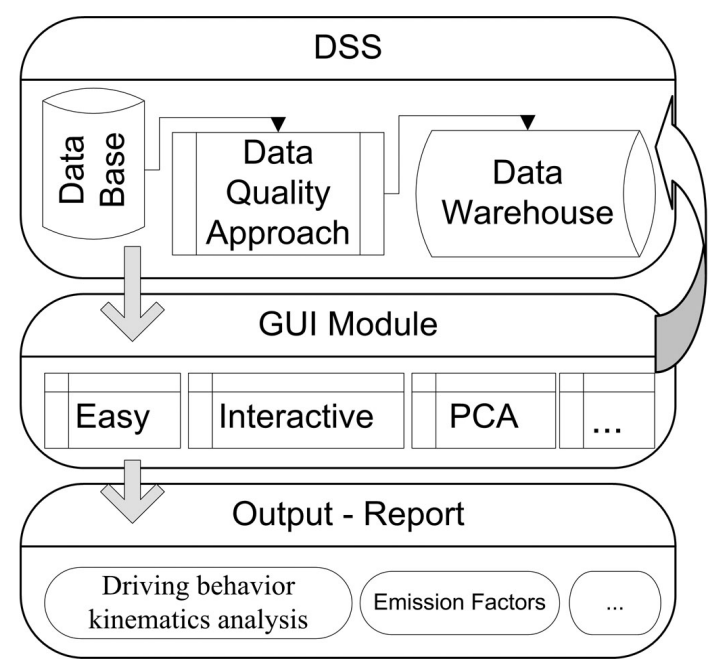

Figure 1. IS structure.

\section{The Data Warehouse}

Starting from the ARTEMIS database, a structured data warehouse is built. In the database, a large number of driving cycles (about 203 cycles divided into 671 sub-cycles) are used for measuring emission factors. In all, 2753 tests are carried out by 11 European laboratories as follows:

- 537 tests to look at the influence of driving patterns

- 1334 tests to look at the influence of vehicle parameters

- 672 tests to look at the influence of laboratory related parameters

- 210 tests are part of the round robin test.

The tests are specifically grouped into 17 task conditions to test several vehicle parameters and laboratory conditions. All tests are performed by 183 different vehicles in terms of fuel and emission standards (pre-Euro 1, Euro 1 to Euro 4) $[15]$.

Making reference to ARTEMIS data base version 20050114, the vehicle fleet is divided into several sets, starting from homologation (Euro 1 to Euro 4) and displacement factors. Three classes of displacement are assumed: 12001400 cc, 1400-2000, over 2000.

To obtain a useful data warehouse, the data quality approach, shown in the following flow chart (Figure 2), is applied. 


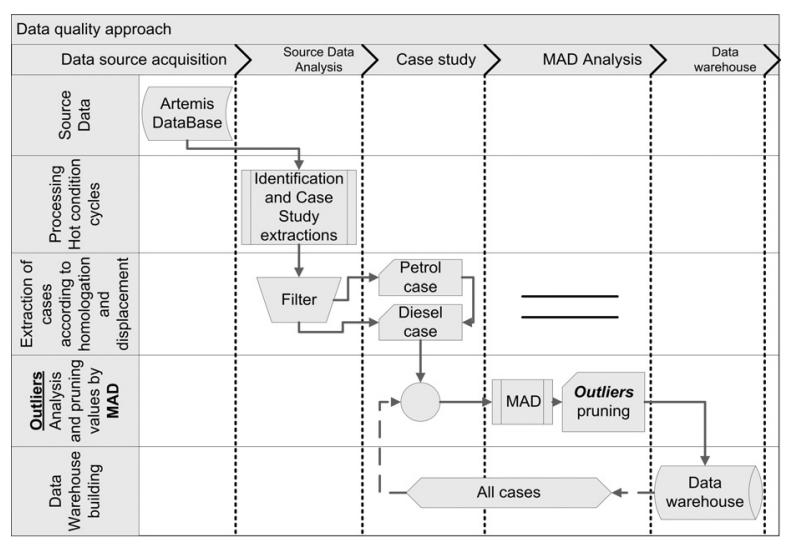

Figure 2. Flow chart of data quality approach.

After the identification of sets, grouping the data starting from homologation and displacement, a median absolute deviation (MAD) approach is used. MAD is a resistant measure of the variability of an univariate sample and, able to identify the presence of one or more outliers. This allows us to identify the presence of outlier values in the data and avoid inserting them in the data warehouse. The procedure is iterated for each case.

Moreover, for each case to consider the effect of different vehicles, a specific PLS model is calculated to evidence characteristic trends in emissions and any abnormal values, through the use of dummy variables. These variables allow evaluation of the individual weights of the vehicle coefficients with respect to the pollutants. By using these coefficients, we can identify the percentage effect of any vehicle on a particular emission factor and establish a cut-off point for vehicles considered normal emitters from high emitters. Finally, vehicles considered as outliers must be identified and excluded from the training process of emission factors [16].

\section{The Core of IS: the Model Base}

As regards the approach developed in the ARTEMIS project, some studies were developed to take driving behavior into account and to link emissions with kinematics. The kinematic approach and traffic situation approach [5-16-17] were adopted to analyze emission data of different combinations of vehicle types and driving behavior. In this approach it is important to stress the large number of kinematic parameters used to characterize the kinematics of driving cycles that lead to a very powerful and flexible emission model, yet which are rather complex in statistical and mathematical terms.

Hereinafter, mathematical aspects and equations of KEM are presented. A consistent set of kinematic parameters is used to represent the real-world driving behavior of vehicles in any traffic situation. In KEM, the response considered is the measured unit emission mass of $\mathrm{CO}$, $\mathrm{HC}, \mathrm{NOx}, \mathrm{CO}_{2}$ (expressed in $\mathrm{g} / \mathrm{km}$ ), measured in a driving cycle (DC), that is a portion of a trip. A $\log$-transform of $Y_{i j}\left(\eta_{i j}=\ln Y_{i j}\right)$ is applied because emission quantities are (in most cases) close to zero with a large coefficient of variation and because emission data are distributed according to a lognormal distribution. The explicative variables characterize the kinematics of driving cycles. These variables are identified by considering emission variation as explained by the variation in exhaust mass (function of energy spent by the vehicle in a driving cycle), and the frequency of acceleration events at different speeds. Two blocks of variables are then associated to the different aspects: the first is formed by variables related to the dynamic vehicle equation, plus idling time to consider stand-still phase emission production. The second block of 42 variables summarizes kinematic acceleration events in DC, whose distribution especially affects $\mathrm{CO}, \mathrm{HC}$ and NOX emissions. Also, speed/acceleration distribution is utilized in ARTEMIS to analyze and determine driving cycle features.

Because of collinearity of variables highly correlated with each other in different groups representing different kinematic aspects of different DCs, Principal Component Analysis and the Partial Least Squares (PLS) Regression [17-1819] based on PCA variables statistical methods are implemented in IS. The NIPALS algorithm is applied to estimate the regression model. Then the following two regression equations are defined for the two blocks:

$$
\begin{aligned}
\ln Y_{i j} & =a_{0}+a_{1} M V+a_{2} M V 2+a_{3} M V 3 \\
& +a_{4} M V A \_P O S+a_{5} \text { Trunning } \\
& +a_{6} \text { Tidle }+a_{7} \text { Invdist }+\varepsilon_{i j}
\end{aligned}
$$

where $M V$ is mean speed, $M V 2$ is mean square speed and $M V 3$ is mean cubic speed; $M V A \_P O S$ is the mean product of instantaneous speed $(v)$ and acceleration $(a)$, calculated for positive values of $a$ and $v$; Trunning is the running time and 
Tidle is the idle time of the driving cycle; Invdist is the reciprocal of the trip length and $a_{1}-a_{7}$ are the regression coefficients;

$$
\begin{aligned}
\ln Y_{i j} & =b_{0}+b_{1} F S_{-} V 20 a 1+b_{2} F S_{-} V 20 a 2 \\
& +\ldots+b_{42} F S_{-} V 101 a 7+\varepsilon_{i j}
\end{aligned}
$$

where $F S_{-} V_{i} a_{j}$ for $i=1, \ldots, 6, j=1, \ldots, 7$ is the centered log-transform of cycle time frequency in the cell determined by six classes of speed and seven classes of acceleration, and $b_{1-}$ $b_{42}$ are the regression coefficients.

In both equations random noise $\varepsilon$ is assumed to be a normally distributed random variable, $\varepsilon \sim N\left(0, \sigma^{2}\right)$.

According to a multi-block approach (MBPLS) [5], a base model 1 is defined for block 1 and a base model 2 is calculated for block 2 of variables. Then the super block regression model, named top model, is built, making the regression of Y-variables on the union of scores of the two base models.

The equation is:

$$
\begin{aligned}
\ln Y_{i j} & =C_{1} M 1 . T_{1}+C_{2} M 2 . T_{2}+\ldots+C_{k^{\prime}} M 1 . T_{k^{\prime}} \\
& +C_{k^{\prime}+1} M 2 . T_{1}+C_{k^{\prime}+2} M 2 . T_{2} \\
& +\ldots+C_{K} M 2 . T_{k^{\prime \prime}}
\end{aligned}
$$

where $T_{1} \ldots T_{k^{\prime}}$ and $T_{k^{\prime}+1}, \ldots T_{k^{\prime \prime}}$ are the two sets of principal components estimated separately for each block of variables, fitting the two base models previously defined. Thus these super scores are the union of scores of the two base models and this so-called top model estimates the coefficient $C_{K}$ and the predicted values of regression of $\ln Y$ on the set of $X$ variables.

The effect of individual vehicles on the response is assessed by developing a further regression equation made of dummy variables, one for each vehicle. Then a vehicle effect base model is calculated.

Emission factors can be calculated from each of the four models according to the level of analysis and to each specific case study.

It is then necessary to apply a retransformation of $\ln Y$ to obtain emission factors in original scale [20]. The formula used to calculate model expectations is:

$$
\begin{aligned}
& E[\text { p, veh.class }]=\overparen{Y}= \\
& \quad \exp \left[\ln \widehat{Y}+(\text { RMSEE })^{2} / 2\right]
\end{aligned}
$$

Since a log transformation was applied to response variables, quantities predicted by model $[\mathrm{E}(\ln \mathrm{Y})]$ must be retransformed in original scale to get emission factors in original unit $\mathrm{g} / \mathrm{km}$.

\section{The User Interface}

The effectiveness of DSS is assured by a set of tools, and the graphical user interface (GUI) module, that interact with the data warehouse. As the DSS is flexible, it is possible in the future to insert a new level of interaction. As a starting point in the development concept design, we can identify three levels (Figure 3):

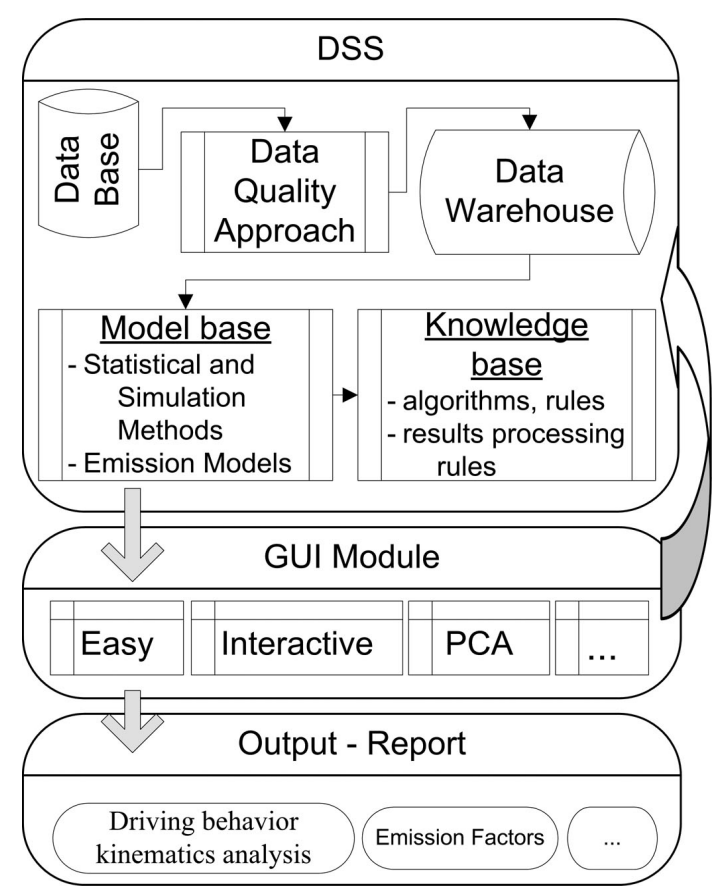

Figure 3. DSS development concept design.

Easy module: able to predict emission factors based on the input of several parameters. The input variables are:

- Driving cycle profile;

- Homologation and displacement;

- Model parameters;

The core of the tool is a set of regression equations applying an empirical model which calculates from the MBPLS approach the vehicle emissions in real-world hot driving conditions. 
Interactive module: able to select several parameters or block information in the data warehouse to rebuild the MBPLS and obtain new regression coefficients to update the DSS and use the new information in the easy module.

PCA module: able to analyze a new set of driving cycles and relative pollution not contained in the historical data warehouse. The tool, based on Principal Component Analysis, provides analysts with the possibility of exploring their own driving cycles, in terms of exploratory and confirmatory emission factor analysis, and updating the database.

\section{Applications and Results}

Application concerns a selected case study, the Euro III gasoline passenger car in the 1200$1400 \mathrm{cc}$ displacement range. Some results of the driving behavior kinematics analysis and predicted emission factors are shown.

Choosing the Easy module, it is possible, by selecting the right parameter input, to obtain several representations of predicted emission factors. In Figures 4 and 5, a 3D surface plot of predicted $\mathrm{CO}_{2}$ and $\mathrm{HC}$ in $\mathrm{g} / \mathrm{km}$ on first two scores is shown. In agreement with principal components, predicted $\mathrm{CO}_{2}$ values are higher for urban and motorway DC than for rural.

By selecting the PCA module it is possible, in terms of Principal Component Analysis, to study the driving behavior kinematics of all driving cycles. The relation of principal components $(\mathrm{PC})(\mathrm{t} 1, \mathrm{t} 2)$ with observations (driving cycles), is shown in Figure 6. These scores are new variables computed as a linear combination of all the original variables to provide a good summary. This graphical representation shows the kinematics distribution of driving behavior and the possible presence of outliers, groups or other patterns in the data. This kind of analysis is useful to explore new DCs with respect to the database and also to improve and update it. In Figure 6 we can identify three groupings of DCs: urban, rural and motorway. This distribution depends on the original variables that were used to build the model. In fact the first PC is positive related with slow DC with high Invdist and high Tidle. The second PC is positive related with low and constant level of acceleration at high speed.

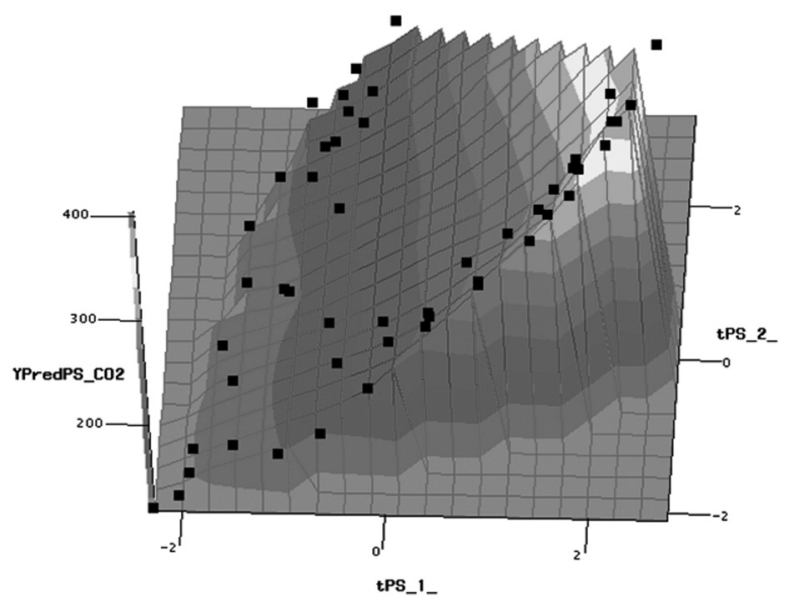

Figure 4. 3D surface plot of predicted $\mathrm{CO}_{2}$ (YPredPS) in $\mathrm{g} / \mathrm{km}$ on tPS_1 vs tPS_2 score.

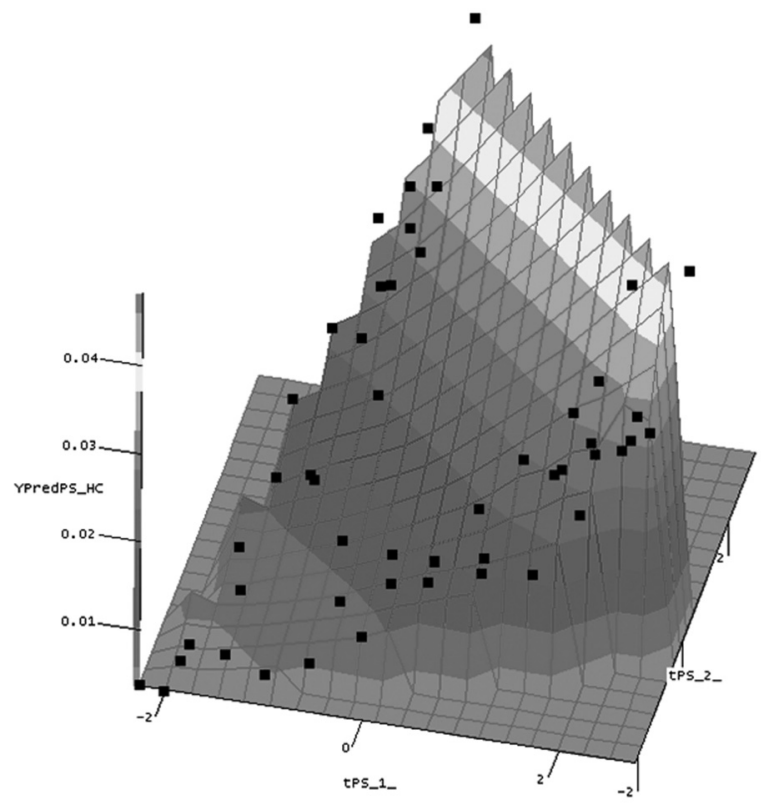

Figure 5. 3D surface plot of predicted HC YPredPS) in $\mathrm{g} / \mathrm{km}$ on tPS_1 vs tPS_2 score.

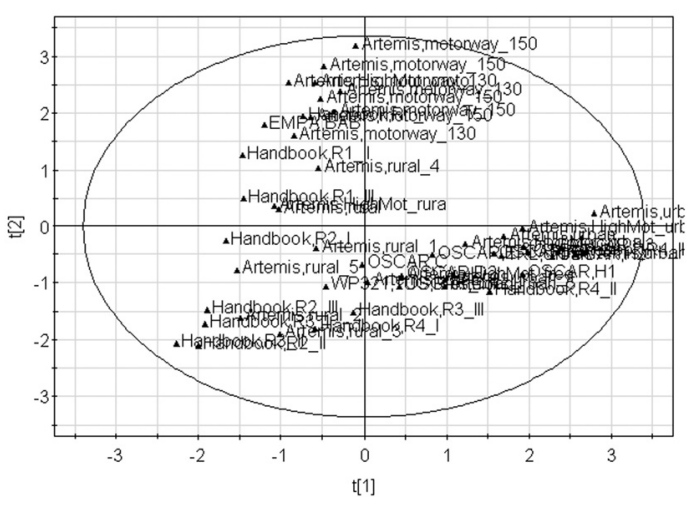

Figure 6. Score plot of t1 vs t2. 


\section{Conclusion}

A comprehensive modelling approach was developed and validated by statistical methods, as well as by comparison with other models.

A DSS was designed to transform a modelling approach into an effective real-world emission analysis and predicting tool, both for research and for policy making.

The DSS components available are a large Emission Data Base and the Model computing modules. The flexibility of the DSS allows new levels of interaction to be inserted in the IS.

Automation of computing processes requires the implementation of a Data Warehouse and a Model Base. The most strategic task is the elaboration of a graphical user interface (GUI) for the production of reports. This is the tool of DSS which interacts with the user, to make a query and receive a response.

A minimum example of DSS potential is shown by the output relative to a case study. The full development of DSS is in progress.

\section{Acknowledgments}

Experimental data reported in the paper were obtained in the research work carried out within the EU Artemis project and under a convention between Istituto Motori and the Italian Ministry of the Environment and Land Conservation (MATT).

\section{References}

[1] R. A. Giannelli, S. SRivastava, G. Dolce, Sensitivity Analysis of MOBILE6.0, EPA420-R-02-035, 2002 .

[2] EMFAC2007 Calculating emission inventories for vehicles in California http://www.arb.ca.gov /msei/onroad/downloads/docs/user_guide_ emfac2007.pdf

[3] LEONIDAS NTZIACHRISTOS, CHARIS KOURIDIS, SAVAS GEIVANIDIS, ATHANASIOS SAMARAS ZISSIS MAMAKOS, COPERT 4 Methodology and Software Updates, LAT Aristotle University Thessaloniki; 2006.

[4] R. Joumard, J. M. ANDRE, M. RAPONE, Emission factor modelling and database for light vehicles Artemis deliverable 3, 2007.
[5] M. Rapone, M.V. Prati, M.A. Costagliola, L. Della Ragione, G. MecCARIELlo, Emission Factors Determination of Euro III 1,200- to 1,400-cc Petrol Passenger Cars with a PLS Multivariate Regression Model, Journal Environmental Modelling and Assessment, Springer Netherlands, ISSN 14202026 (Print) 1573-2967 (Online).

[6] R. SMit, R. SMokers, E. SCHOEN, VERSIT+LD Development of a new emission factor model for passenger cars linking real world emissions to driving cycle characteristics, 14th International Conference Transport and Air Pollution, 2005.

[7] D. Schulz, T. Younglove AND M. BARTH, Statistical Analysis and Model Validation of Automobile Emissions, Journal of Transportation and Statistics, 2000

[8] M. Barth, F. An, T. Younglove, G. Scora, C. LEVINE, CMEM Comprehensive Modal Emissions Model User's Guide version 2.0, NCHRP Project 25-11, January 2000

[9] M. Barth, C. Malcolm, N. Hill, T. Younglove, Recent Validation Efforts for a Comprehensive Modal Emissions Model, Transportation Research Board 80th Annual Meeting, 2001

[10] M. BARTh, J. COLlins, G. SCORA, N. DAVIS, J. NORBECK, Measuring and Modelling Emissions from Extremely Low Emitting Vehicles, Transportation Research Record (Transp. Res. Rec.) ISSN 03611981 CODEN TRREDM , Air quality 2006

[11] M. Barth, T. Younglove, C. Malcolm, G. SCORA, Mobile Source Emissions New Generation Model: Using A Hybrid Database Prediction Technique, U.S. Environmental Protection Agency $A S D$ 01-TS-26655-02-DFR, February 2002

[12] T. J. BARlow, P. G. Boulter, I. S. McCrae, Scoping study on the potential for instantaneous emission modelling: summary report, TRL framework contract no. 3/323-R041, 2007.

[13] R. Engelmore, T. Morgan, Blackboard systems, Addison - Wesley, Wokingham, England, 1988

[14] B. Shmulyian, A. Fedotov, L. Heifits, Sh. IMELBAYEV, G. KIRSANOVA, Information technology for urban land-use and transport analysis: principle and data-handler. AIRO'90 Conference, Sorrento, Italy, 1990

[15] R. JOUMARD, M. ANDRÉ, J. LAURIKKO, T. LE ANH, S. GEIVANidis, Z. SAMARAS, Z. OlÁH, P. DEVAuX, J. M. ANDRÉ, E. CORNELIS, P. ROUVEIROLlES, S. LACOUR, M. V. PRATI, R. VERMEULEN, M. ZALLINGER, Accuracy of exhaust emissions measurements on vehicle bench (Artemis deliverable 2), INRETS report, Bron, France, Report no. LTE 05222006.

[16] M. Rapone, M. V. Prati, G. Meccariello, L. Della Ragione, M. A. Costagliola, A Novel Statistical Model For The Evaluation of Vehicle Emission Factors. Application to a Euro III Gasoline Car Fleet, Proceedings of the 7th International Conference on Engines for Automobile. Ice2005. Capri, Naples. September 2005. Sae Paper 200524-024. 
[17] M. TenEnhaus, La regression PLS Theorie et Pratique, Editions Technip Paris, 1998.

[18] S. Wold, M. SJostrom, L. EriksSON, PLSregression: a basic tool of chemometrics, Chemometrics and Intelligent Laboratory Systems 58, 2001, pp. 109-130.

[19] J. A. Westerhuis, T. Kourti, J. F. MacGregor, Analysis of Multiblock and Hierarchical PCA and PLS Models, J. of Chemometrics; 1998, 12, pp. 301-321.

[20] N. DUAN Smearing Estimate, A nonparametric retransformation method, Journal of American Statistical Association, September 1983, pp. 78-383.

Received: June, 2008 Accepted: September, 2008

Contact addresses:

Mario Rapone $\dagger$ Istituto Motori CNR

via Marconi 8

80125 Naples Italy

Fax +390812396097

e-mail: m.rapone@im.cnr.it

Livia Della Ragione

Istituto Motori CNR

via Marconi 8

80125 Naples Italy

Fax +390812396097

e-mail: 1.dellaragione@im.cnr.it

Giovanni Meccariello

Istituto Motori CNR

via Marconi 8

80125 Naples Italy

Fax +390812396097

e-mail: g.meccariello@im.cnr.it
DR. MARIO RAPONE ${ }^{\dagger}$ graduated in mechanical engineering (University of Naples). His experience is in statistical modeling application to engineering with particular concern to transportation systems. Major topics regard Transportation System reliability and environmental impact. Recent scientific results regard experimental detection and modeling of real use vehicle performance and emissions, with reference to passenger cars and buses.

DR. LIVIA DELLA RAGIONE graduated in 1988 in mathematics from the University of Naples "Federico II" (Italy). Currently, she is a researcher of CNR-IM. Her main research activities concern the environmental impact of vehicles. Her principal areas of interest include multivariate statistical analysis and time series analysis. The main research subjects are vehicle mission profile in urban areas and the development of methods for the evaluation of exhaust emissions as a function of vehicle operating conditions in the traffic. In this context she has developed some statistical codes applied to determine driving cycles by vehicle speed records in the traffic obtained by instrumented cars performing planned. Also, she is involved in the development of models for vehicular emission factors determination based on measurements of pollutant emissions.

Dr. Della Ragione is responsible of research group working on "Statistical methods for driving cycle modelling and emission factors development".

DR. GIOVANNI MECCARIELLO graduated in economics from the University of Naples "Federico II". He is a researcher in the vehicle performance and emissions for sustainable transportation at Istituto Motori of National Research Council of Italy. His research field of interest includes statistical and soft computing methods for modeling driving cycles and emissions of vehicles in real-use with special concern to vehicle emission profile in urban areas and exhaust emissions evaluation. He is working with SAS System, particularly in the field of multivariate statistical analysis with SAS/STAT ${ }^{\circledR}$ and SAS/GRAPH®. 